\title{
MICRO HEAT EXCHANGER BY USING MEMS IMPINGING JETS
}

\author{
S. Wu, J. Mai*, Y.C. Tai, and C.M. Ho ${ }^{*}$ \\ Electrical Engineering 136-93, California Institute of Technology, Pasadena, CA 91125 \\ * MAE Dept., UCLA, Los Angeles, CA 90024
}

\begin{abstract}
A micro impinging-jet heat exchanger is presented here. Heat transfer is studied for single jet, slot arrays and jet arrays. In order to facilitate micro heat transfer measurements with these devices, a MEMS sensor chip, which has an $8 \times 8$ temperaturesensor array on one side, and an integrated heater on the other side has been designed and fabricated. This sensor chip allows 2-D surface temperature measurement with various jets impinging on it. It is found that micro impinging jets can be highly efficient when compared to existing macro impinging-jet microelectronics packages such as IBM 4381. For example, using a single nozzle jet $(500-\mu \mathrm{m}$ diameter driven by 5 psig pressure), the sensor chip $\left(2 \times 2 \mathrm{~cm}^{2}\right)$ temperature can be cooled down from 70 to $33^{\circ} \mathrm{C}$. The cooling becomes more efficient when nozzle arrays (4x5 over $1 \mathrm{~cm}^{2}$ area) are used under the same driving pressure. Interestingly, although higher driving pressure gives better cooling (lower surface temperature), the cooling efficiency, defined as $h / 0.5 \rho v^{2}$, is actually higher for lower driving pressure.
\end{abstract}

\section{INTRODUCTION}

The thermal conductivity of thin films can be one to two orders of magnitude lower than the bulk thermal conductivity [1]; thus even a small power dissipation in a thin-film region may cause serious local heating in micro devices including IC's and MEMS. Moreover, as the transistor density and/or the number of transistors on a standard-sized chip, increases in IC's, the power dissipation also increases. It is therefore necessary to investigate better thermal cooling methods for future chip cooling. It is interesting to note that for a chip under a given heat flux and a maximum allowable chip temperature increase, the tangential forcedconvection heat transfer coefficient, $h$, is on the order of $0.01 \mathrm{~W} / \mathrm{cm}^{2} \mathrm{~K}[2]$. For impinging-jet cooling, on the other hand, the heat transfer coefficient can be an order of magnitude larger, i.e., approximately $h=0.1$ $\mathrm{W} / \mathrm{cm}^{2} \mathrm{~K}$. Thus, impinging-jet cooling is a promising cooling method with the advantage that it is still single-phase (gas phase) cooling.

Nevertheless, impinging-jet cooling experiments encountered in the literature to date have mainly used macro-scale impinging jets [3]. In addition, these works focused largely on turbulent jets with $H / D$ ratios greater than 1.0 , where $H$ is defined as the space between the nozzle and impinged surface (also called height of the jet) and $D$ as the diameter of the jet. What is interesting is that the regime with $H / D$ ratios less than 1.0 now can be studied since MEMS technology can produce micron-scaled jets. As a result, a more efficient micro heat exchanger may become possible as micro jets can be placed much closer to the hot surface than macro jets. The goal of this work is then to study micro impinging jet cooling, focusing on experimentation with variable parameters of height, nozzle diameter, and nozzle spacing in the sub-millimeter range.

\section{DESIGN AND FABRICATION}

\section{Temperature Sensor Chip}

A specialized temperature-sensor-array chip, shown in Figure 1, was fabricated in order to serve as a test target for the impinging jets. This $2 \mathrm{~cm} \times 2 \mathrm{~cm}$ chip has an integrated polysilicon heater on the backside and $8 \times 8$ temperature sensors on the front side. The 64 thermistor-type polysilicon sensors, each $4 \mu \mathrm{m} \times 4 \mu \mathrm{m}$, are $500 \mu \mathrm{m}$ apart and are placed in a $4 \mathrm{~mm} \times 4 \mathrm{~mm}$ area at the center of the top surface. These polysilicon temperature sensors have a nominal room temperature resistance of about $20 \mathrm{k} \Omega$ and a temperature resolution of $0.1^{\circ} \mathrm{C}$.

The fabrication process of the sensor chip is given in Fig. 2. Starting with bare $<100>$ silicon, a $5000 \AA$ thick layer of dry thermal oxide and a $2000 \AA$ thick layer of LPCVD nitride are deposited. Then $5000 \AA$ of LPCVD polysilicon is deposited for the sensors and doped with boron to a concentration of 5 $x 10^{18} \mathrm{~cm}^{-3}$. The sensors are then encased in another $2000 \AA$ thick nitride layer that electrically isolates 
and protects the sensors. Finally contact holes are opened in the nitride by RIE and aluminum metallization finishes the sensor process. Here, the backside heater is fabricated similarly, with the only differences in masking and the backside polysilicon doping, i.e., heavily boron doped at $5 \times 10^{20} \mathrm{~cm}^{-3}$.

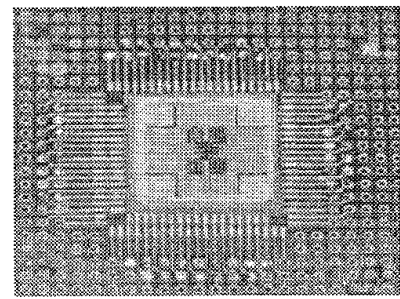

Figure 1. Temperature-sensor chip mounted on a $\mathrm{PCB}$ in a Plexiglas chuck.

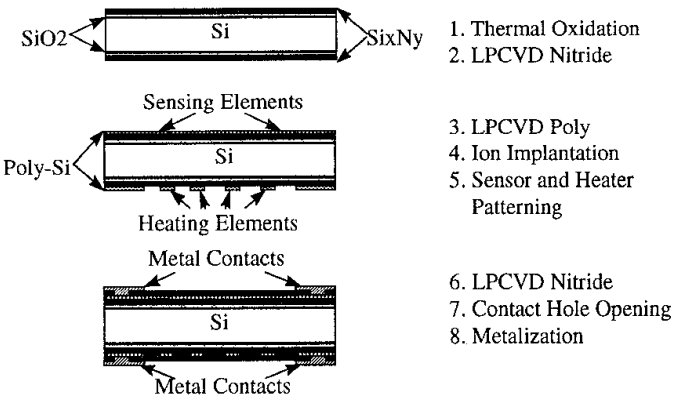

Figure 2. Process flow for a temperature-sensor chip with an integrated heater on the backside.
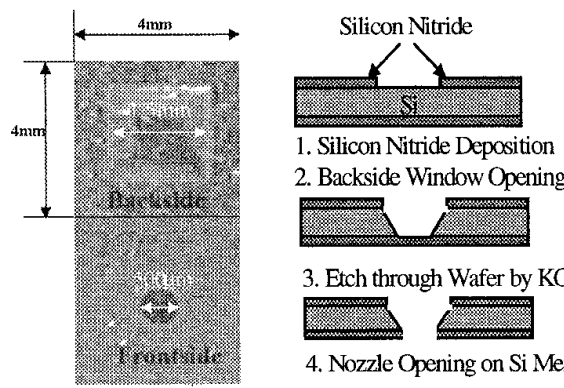

3. Etch through Wafer by $\mathrm{KOH}$

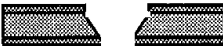

4. Nozzle Opening on Si Membrane

Figure 3. MEMS single nozzle chip and its fabrication process.

\section{MEMS Single Nozzle}

Fig. 3 shows a fabricated MEMS single nozzle chip and its fabrication process. It is fabricated on a $3000 \AA$ thick nitride layer deposited on both sides of $a<100>$ Si wafer. The gas-inlet square holes on the backside, are formed by $\mathrm{KOH}$ etching through the (500 $\mu \mathrm{m}$ thick) wafer, and a circular nozzle hole is defined on the front nitride diaphragm using RIE. This process is used to produce circular single jets with nozzle diameters ranging from 0.5 to $1.5 \mathrm{~mm}$.

\section{MEMS Nozzle/Slot Arrays}

MEMS nozzle and slot arrays have also been fabricated. These arrays are made by bonding two separate chips together. The fabrication process of both chips is shown in Fig. 4. The wafer on the left defines the nozzles/slots and the wafer on the right defines the gas inlets. The two pieces are fabricated with the same process. Starting with $\langle 100\rangle \mathrm{Si}$ wafers, openings for the nozzles/slots are first etched in $\mathrm{KOH}$. Then a $5000 \AA$ thick thermal oxide is grown on both sides over the openings as a protective layer. Etching windows are then opened in the oxide, and followed by $\mathrm{KOH}$ through-wafer etching. After dicing, the thermal oxide is removed using BHF. To make a final array chip, a pair of matching chips are then aligned and fusion bonded. Finally, the bonded array chip is deposited with a $4-\mu \mathrm{m}$-thick layer of Parylene. This Parylene layer serves two purposes. One is to seal any unbonded surfaces, and the other is to increase thermal insulation from ambient.

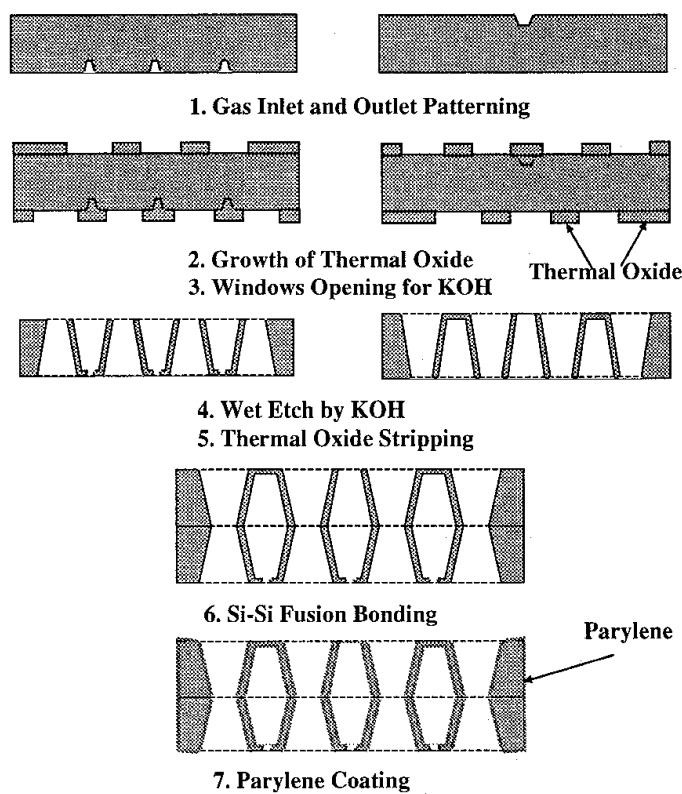

Figure 4. MEMS jet array process flow.

Fig. 5 shows the finished nozzle and slot arrays. In the figure, the circular nozzles are $1 \mathrm{~mm}$ diameter, and the slots are $1 \mathrm{~mm}$ wide and $8 \mathrm{~mm}$ long. Rows of nozzles/slots are spaced $2.5 \mathrm{~mm}$ apart from center to center. The entire structure is approximately $1 \mathrm{~mm}$ thick. 

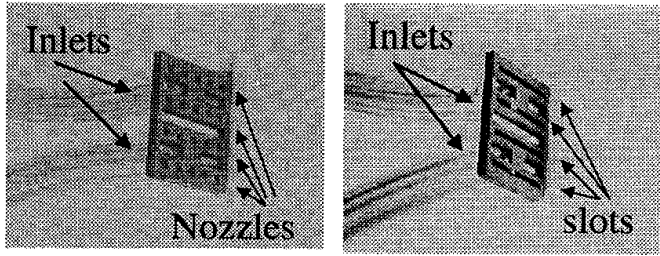

Figure 5. Micromachined nozzle and slot array.

\section{EXPERIMENTAL SETUP}

Fig. 6 shows the experiment a setup. The chip is mounted on a Plexiglas chuck for thermal isolation. The backside polysilicon heater has a nominal resistance of $55 \Omega$, and by varying the input voltage, we have a precisely controllable heat source using the Joule heating of this resistor. Most tests are conducted with approximately $1 \mathrm{~W}$ of power. As shown, a vacuum is always drawn on the backside of the chip to prevent downward heat loss. This is important because it's desirable to have all the heat transferred to the top surface. Above the sensor chip, filtered air is applied to drive an impinging-jet nozzle or array chip, which is then held above the sensor chip by an aluminum L-block that is attached to a 3axis micro-positioner. The inlet air/gas system has sensors and valves to monitor and control the inlet pressure and the mass flow rate.

Each sensor on the chip is wire bonded to leads on a PCB mounted on the top surface of the Plexiglas chuck. The PCB is connected to a HP 34970A data acquisition system that has been set up to measure resistance. An environmental chamber is used to obtain sensor temperature calibration curves, which are fitted by a second-order polynomial, are used for data processing. These sensors are calibrated from room temperature to $80^{\circ} \mathrm{C}$.

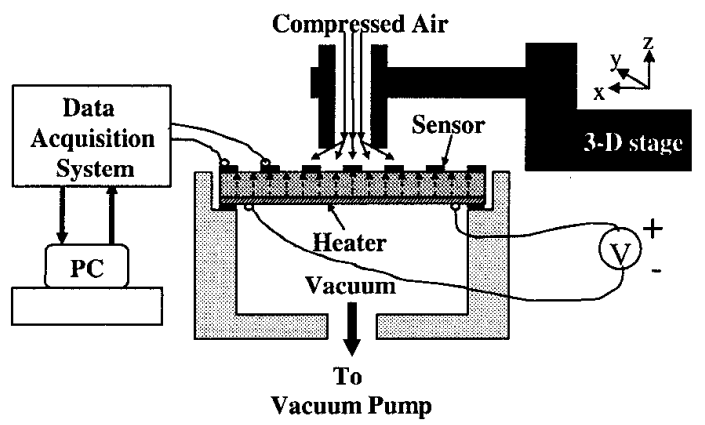

Figure 6. Experimental Setup.

\section{RESULTS}

A single glass nozzle, a MEMS single nozzle, a MEMS nozzle array and a MEMS slot array were tested at heights ranging from 100 to $3000 \mu \mathrm{m}$, and with pressures ranging from 0.5 to $5 \mathrm{psig}$.

\section{Temperature Distribution Measurement}

Temperature distribution measurements must be performed first to confirm the functionality of the sensor chip by using a MEMS single jet which is 750 $\mu \mathrm{m}$ away from the sensor chip and driven by 2 psig compressed air. Fig. 7 shows some typical data imaged in this fashion. The sensor chip must be able to identify and track the jet as it is moved around as in Fig. 7. Otherwise, the chip does not function properly.

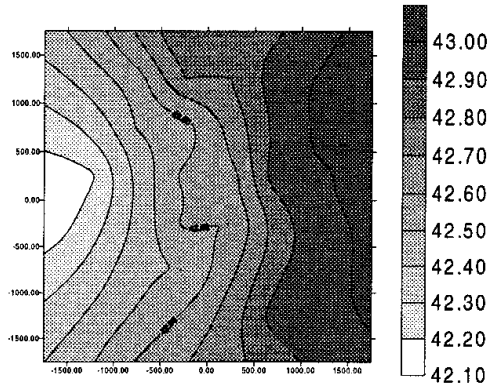

(a) Jet on the left

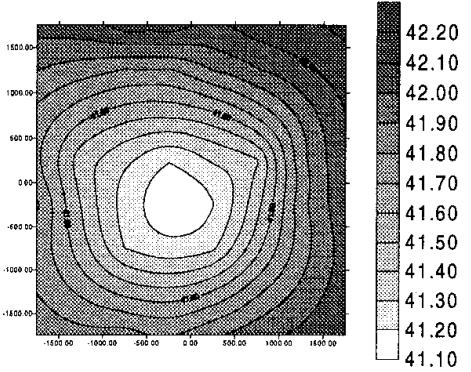

(b) Jet at center

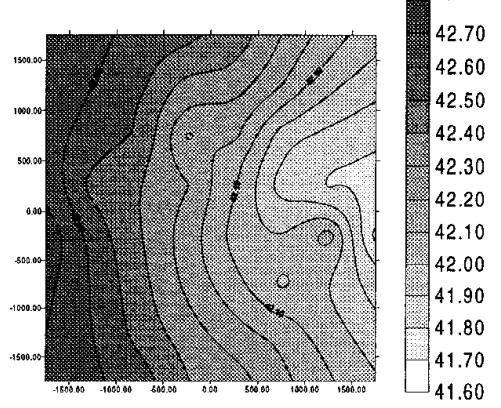

(c) Jet on the right

Figure 7. Contour temperature plots indicating that the sensor image correctly identifies and tracks an impinging jet. 


\section{Single Impinging Jet Cooling}

Fig. 8 shows three sensor chip temperature profiles measured under three conditions. First, the temperature profile at the bottom was measured at room temperature without any heating and cooling. This step actually serves to confirm the reliability of our sensor chip. The uniformity $\left( \pm 0.1{ }^{\circ} \mathrm{C}\right)$ of the measured temperatures shows good system reliability over a span of a few days. Then, the top temperature distribution profile is measured with $1.12 \mathrm{~W}$ heater power consumption, but only with natural convection on frontside. Finally, the middle profile is the temperature under the cooling of a $500 \mu \mathrm{m}$ diameter MEMS nozzle jet that is $750 \mu \mathrm{m}$ above the surface and driven by 5 psig compressed air. The large temperature drop $\left(>35{ }^{\circ} \mathrm{C}\right)$ from the natural convection profile depicts the effectiveness of the impinging jet.

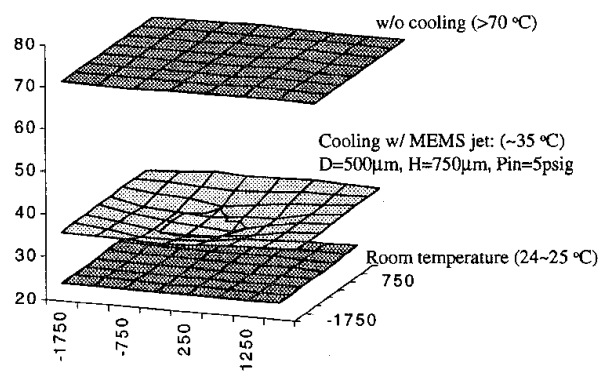

Figure 8. Temperature profiles showing the effectiveness of impinging jet cooling with a single nozzle

Here, we investigate height and pressure effects. Figs. 9 and 10 show the average temperature vs. height of the nozzle under different air pressures, but with the same backside heater power of $1.12 \mathrm{~W}$. The averaged surface temperature of the glass-capillary jet does not vary much even with different heights (Fig. 9). We believe this is due to the fact that the free jet can easily entrain local cold air and circulate it over the hot surface. In the case of the MEMS jet, it is more of a confined jet situation. Since the front surface of the MEMS nozzle is large ( $4 \mathrm{~mm}$ by 4 $\mathrm{mm}$ ), extra flow resistance will be induced at $H<I \mathrm{~mm}$, hence cooling capability will be decreased. At larger heights, this effect is not observed and the MEMS jet has a cooling capability comparable to the free jet (shown in Fig. 10). Here, the heat transfer coefficient for the free jet can be calculated to be $0.032 \mathrm{~W} / \mathrm{cm}^{2} \mathrm{~K}$ at $5 \mathrm{psig}$ and $0.025 \mathrm{~W} / \mathrm{cm}^{2} \mathrm{~K}$ at $3 \mathrm{psig}$, assuming that the single jet has an effective area of the whole chip. If the effective area of the jet is smaller than the chip area, the heat transfer coefficient should be much larger. Therefore, the calculated values are really the lower bounds of the heat transfer coefficients. Nevertheless, we can conclude from Figs. 9 and 10 that cooling capability of a single jet increases with its driving pressure, and the MEMS jet has similar cooling characteristics to a free jet when the $H / D$ ratio is greater than 3 . Comparison of the Reynolds number indicates the observed results agree with the expected flow regime. The Reynolds number for $H / D<1$ with the MEMS jet should scale with the height, which is now the minimum opening in the system. This yields values of the Reynolds number as low as 1300 , which is in the lower end of the transition region, but near laminar flow. In this regime, it is known to have poor heat transfer characteristics.

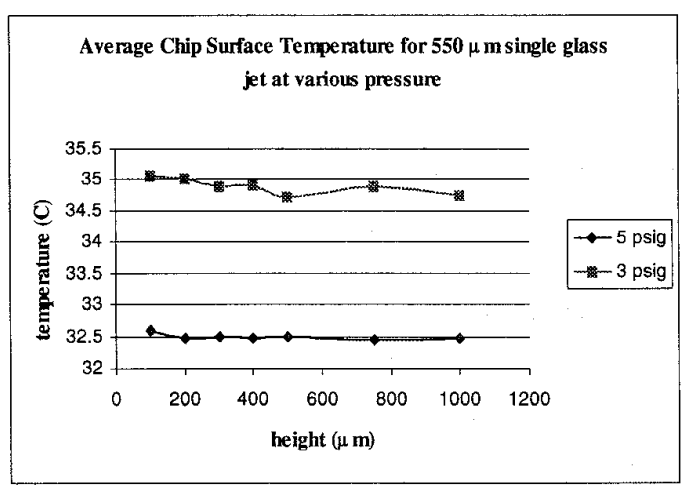

Figure 9. Average sensor chip temperature at various heights for two inlet pressures of a free jet.

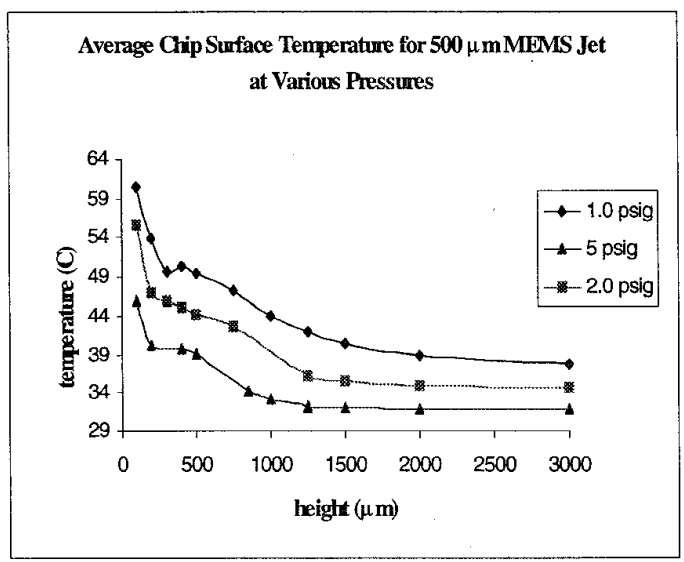

Figure 10. Average chip temperature at various heights for a $500 \mu \mathrm{m}$ diameter nozzle jet.

Jet cooling efficiency, defined as heat transfer coefficient normalized by the kinetic energy of gas (i.e., $h / 0.5 \rho v^{2}$ ), has been calculated and plotted in Figs. 11 and 12, where Fig. 11 is for a single free jet and Fig. 12 is for a MEMS jet. It is clear that for both 
types of jets, lower driving pressure gives a higher cooling efficiency. This brings up an important issue; that is the design of impinging jet cooling depends on the gas sources. For example, if a constant pressure source is available without the worry of running out of gas, then the higher the pressure, the better. On the other hand, if the gas source has a limited amount of gas, higher efficiency, i.e., lower pressure, should be considered.

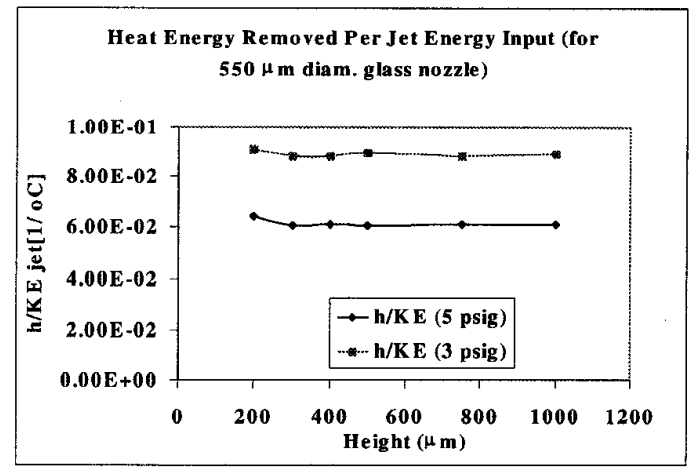

Figure 11. Cooling efficiency at various heights at two inlet pressures of a free single jet

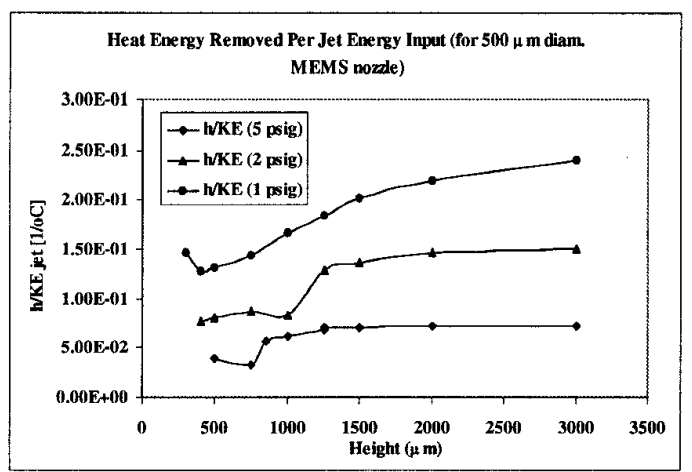

Figure 12. Cooling efficiency at various heights at three inlet pressures of a MEMS single jet

\section{Jet Array Cooling}

Nozzle array and slot array chips were also tested with the same setup as single jets. Figs. 13 and 14 show examples of the temperature distribution measured from array chips. First, the temperature images do not show same distinctive jet boundaries as those in the images of single jets. This may be due to a more complicated jet flow pattern. However, it is noted that the temperature distribution is more uniform than single-jet cooling. This is more evident in the case of nozzle array cooling, where less than $0.25^{\circ} \mathrm{C}$ temperature variation across the whole sensor area is observed.

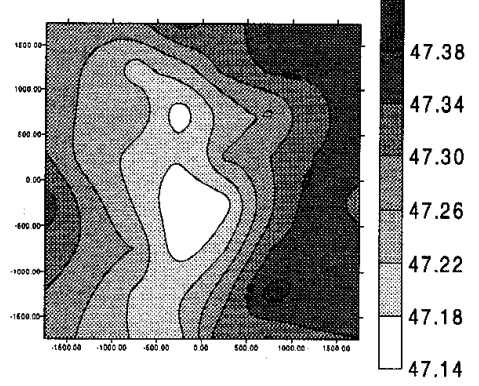

Figure 13. Temperature distribution recorded from a nozzle jet array at $500 \mu \mathrm{m}$ height, $\mathrm{Pin}=$ $0.5 \mathrm{psig}$, and power $=1.12 \mathrm{~W}$.

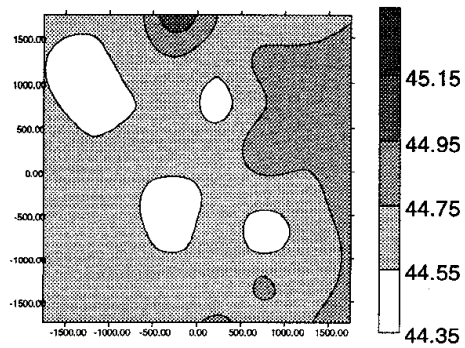

Figure 14. Slot array temperature distribution at height $=1000 \mu \mathrm{m}$, Pin $=1 \mathrm{psig}$, and an input power of $1.12 \mathrm{~W}$.

Finally, it is also interesting to compare cooling effectiveness for various jet conditions by varying the inlet driving pressure to fix a heated surface temperature. This experiment was performed and the summary data is presented here in Table 1 for four different conditions.

\begin{tabular}{|l|l|l|l|l|}
\hline & $\begin{array}{l}\text { Single } \\
\text { MEMS } \\
\text { Jet }\end{array}$ & $\begin{array}{l}\text { Single } \\
\text { Glass } \\
\text { Jet }\end{array}$ & $\begin{array}{l}\text { Slot } \\
\text { Array }\end{array}$ & $\begin{array}{l}\text { Nozzle } \\
\text { Array }\end{array}$ \\
\hline $\begin{array}{l}\text { Gas } \\
\text { Pressure } \\
\text { (psig) }\end{array}$ & 2.0 & 1.0 & 0.7 & 0.5 \\
\hline
\end{tabular}

Table 1. Driving pressures required to cool the test chip surface temperature to $47^{\circ} \mathrm{C}$ with the power dissipation $Q=1.12 \mathrm{~W}$ and $H=500 \mu \mathrm{m}$.

From Table 1, it is clear that the nozzle jet array is the most efficient arrangement among the four variations. Thus, if the available pressure is low, the MEMS nozzle arrays are desirable.

\section{CONCLUSION}

MEMS impinging jet cooling has been investigated for both single jets and jet arrays. A specially designed temperature sensor chip integrated with a polysilicon heater on the backside facilitates 
various micro-cooling experiments. It is found that a micro impinging jet can provide effective cooling. Higher driving pressure gives better cooling, but lower efficiency. This tradeoff should be considered when using MEMS impinging-jet heat exchangers.

\section{ACKNOWLEDGEMENTS}

This work was sponsored by the Air Force Office of Scientific Research, USAF, under grant/contract number 49620-96-1-0376. The authors would like to thank Trevor Roper, Ronghua Zhang, Charles Grosjean, and Qiao Lin for their contribution to the work.

\section{REFERENCES}

[1] Tien, C-L, Majumdar, A., Gerner, F., ed. Microscale Energy Transport Series in Chemical and Mechanical Engineering, Taylor \& Francis, 1998.

[2] Hollworth, B., Durbin, M., "Impinging Cooling of Electronics," J. Heat Transfer, v. 114. p.607-613, August 1992

[3] Martin, H., "Heat and Mass Transfer between Impinging Gas Jets and Solid Surface," Advances in Heat Transfer, v. 13, 1997 